\title{
STUDY OF THE BESSY II BEAM LIFETIME *
}

\author{
S. Khan ${ }^{\dagger}$, BESSY, Berlin, Germany
}

\section{Abstract}

This paper presents beam lifetime measurements at the BESSY II storage ring and their interpretation.

\section{INTRODUCTION}

The beam lifetime is one of the key parameters of a highbrilliance synchrotron radiation source [1] [2]. Commissioning of the BESSY II storage ring in Berlin-Adlershof started in April 1998 [3]. At the time of writing (February 1999), the vacuum vessel was baked out in 3 of 8 sections at $120^{\circ} \mathrm{C}$. During the commisioning period, major parts of the chamber had to be vented several times in order to install new components, and the vacuum is still in a transitory state. It is nevertheless desirable to understand the factors that will ultimately limit the beam lifetime once the vacuum has improved.

Presently, the beam lifetime is clearly dominated by residual gas scattering. Elastic (Coulomb) scattering on the gas nuclei excites betatron oscillations that may exceed the aperture, while inelastic scattering (Bremsstrahlung) on nuclei and electrons leads to momentum loss. Off-momentum electrons can exceed the momentum acceptance given by the rf bucket, or may hit the aperture when displaced by dispersion. In addition, a betatron oscillation is excited if the momentum change happens in a dispersive region.

* This work is funded by the Bundesministerium für Bildung, Wissenschaft, Forschung und Technologie and by the Land Berlin.

† Email: khan@bii.bessy.de

Table 1: Symbols used in this text and their actual or typical values. The residual gas density $n$ is related to the gas pressure by $n\left[\mathrm{~m}^{-3}\right]=2.45 \cdot 10^{22} \cdot p[\mathrm{hPa}]$.

\begin{tabular}{|c|l|l|}
\hline$a$ & vertical half aperture & $8 \mathrm{~mm}$ \\
$\beta_{a}$ & beta function at aperture & $5 \mathrm{~m}$ \\
$\bar{\beta}$ & average beta function & $12 \mathrm{~m}$ \\
$c$ & velocity of light & $3 \cdot 10^{8} \mathrm{~m} / \mathrm{s}$ \\
$\Delta p / p$ & momentum acceptance & 0.03 \\
$\gamma$ & Lorentz factor & 3327 \\
$I$ & beam current & $1-100 \mathrm{~mA}$ \\
$n$ & residual gas density & $(\mathrm{s}$. caption $)$ \\
$N$ & particles per bunch & $10^{9}-10^{10}$ \\
$r_{e}$ & classical electron radius & $2.8 \cdot 10^{-15} \mathrm{~m}$ \\
$\sigma_{x}$ & rms horizontal beam size & $100-300 \mu \mathrm{m}$ \\
$\sigma_{y}$ & rms vertical beam size & $10-50 \mu \mathrm{m}$ \\
$\sigma_{z}$ & rms bunch length & $5-10 \mathrm{~mm}$ \\
$\sigma_{x^{\prime}}$ & rms hor.angular spread & $20-60 \mu \mathrm{rad}$ \\
$Z$ & residual gas atomic number & 7 \\
\hline
\end{tabular}

Once the vacuum has further improved by beam cleaning and bake-out, the Touschek effect will gain importance: when two electrons within a bunch collide, one acquires and the other looses momentum with the same consequences as described above.

The aim of this work was to obtain a consistent picture of the observed beam lifetime under variation of several parameters. As it turns out, the inclusion of other effects (quantum lifetime, ion-related beam loss, etc.) is not required to interpret the data presented below.

\section{THEORY}

In this section, the relations used in the analysis are reviewed. A list of symbols is given in table 1 .

The beam lifetime $\tau$ is defined by the current decay rate $1 / \tau=-\dot{I} / I$, which is the sum of the Touschek $(\mathrm{T})$ rate and the gas scattering $(\mathrm{G})$ rate

$\frac{1}{\tau}=\frac{1}{\tau_{\mathrm{T}}}+\frac{1}{\tau_{\mathrm{G}}}=\frac{1}{\tau_{\mathrm{T}}}+c n\left(\sigma_{\text {elast }}^{\mathrm{N}}+\sigma_{\text {inel }}^{\mathrm{N}}+\sigma_{\text {elast }}^{\mathrm{e}}+\sigma_{\text {inel }}^{\mathrm{e}}\right)$.

The Touschek decay rate can be written as (e.g. [4])

$$
\frac{1}{\tau_{T}}=\frac{N r_{e}^{2} c}{8 \pi \sigma_{x} \sigma_{y} \sigma_{z} \gamma^{2}(\Delta p / p)^{3}} \cdot D\left(\frac{(\Delta p / p)^{2} \sigma_{x^{\prime}}^{2}}{\gamma^{2}}\right)
$$

where $D \approx 0.3$ is a slowly varying function that is evaluated numerically. Relativistic effects and beam polarization modify the Touschek rate on the level of 10-20\% [5].

The total cross sections for elastic and inelastic scattering on residual gas nuclei $(\mathrm{N})$ and electrons (e) are [4]

$$
\begin{aligned}
\sigma_{\text {elast }}^{\mathrm{N}} & =\frac{2 \pi r_{e}^{2} Z^{2}}{\gamma^{2}} \frac{\bar{\beta} \beta_{a}}{a^{2}} \\
\sigma_{\text {inel }}^{\mathrm{N}} & =\frac{4 r_{e}^{2} Z^{2}}{137} \frac{4}{3}\left(\ln \frac{183}{Z^{1 / 3}}\right)\left(\ln \frac{1}{\Delta p / p}-\frac{5}{8}\right) \\
\sigma_{\text {elast }}^{\mathrm{e}} & =\frac{2 \pi r_{e}^{2} Z}{\gamma} \frac{1}{\Delta p / p} \\
\sigma_{\text {inel }}^{\mathrm{e}} & =\frac{4 r_{e}^{2} Z}{137} \frac{4}{3}\left(\ln \frac{2.5 \gamma}{\Delta p / p}-1.4\right)\left(\ln \frac{1}{\Delta p / p}-\frac{5}{8}\right)
\end{aligned}
$$

The distinctly different dependence of Touschek scattering and inelastic gas scattering on the momentum acceptance $\Delta p / p$ (equations 2 and 4 ) can be used to distinguish the two effects by changing the rf voltage (which also changes the bunch length). Coulomb scattering is identified by variation of the physical aperture using scrapers. 


\section{MEASUREMENTS AND RESULTS}

The data shown below were taken in February 1999. The beam lifetime was deduced from the beam current measured by a current transformer. Low currents $(\sim 1 \mathrm{~mA})$ were measured by monitoring synchrotron light with a photodiode [6].

\subsection{Beam Lifetime versus Current}

The gas scattering rate increases with beam current due to synchrotron radiation desorption. However, given a finite pumping rate, the correlation is not necessarily linear and depends on the vacuum history.

The linear dependence of the Touschek rate on the number of particles per bunch (equation 2) is modified by turbulent bunch lengthening and transverse emittance growth due to intrabeam scattering or instabilities.

In view of these uncertainties, the dependence of the lifetime on the beam current was not analysed. Instead, the lifetime was measured as function of rf voltage and aperture within short time intervals at nearly constant current, and the experiments were restricted to $\leq 100 \mathrm{~mA}$.

\subsection{Beam Lifetime versus $R f$ Voltage}

Under the assumption that the momentum acceptance upto $\Delta p / p=0.03$ is determined by the rf bucket and not by aperture restrictions (which was a design constraint for the vacuum chamber [5]), the curves in figure 1 show the lifetime as function of $\Delta p / p$ in the limits of gas scattering and Touschek scattering (solid lines), and different combinations thereof (dashed lines).

The graphs show the lifetime normalized to $\tau_{0}$, the lifetime at $\Delta p / p=0.03$, and do not depend on the actual gas pressure. Some uncertainty remains: (1) The ratio between elastic and inelastic gas scattering depends on $\bar{\beta}$, the average beta function weighted with the local gas pressure. Theoretical pressure profiles suggest values of $\bar{\beta}=10$ $14 \mathrm{~m}$, depending on the beam current. (2) The choice of $Z$ determines the ratio between scattering on gas nuclei and electrons (the latter being of minor importance).

The measured data shown in figure 1 for beam currents between $1 \mathrm{~mA}$ and $100 \mathrm{~mA}$ are consistent with the theoretical expectation. Figure 2 presents the gas scattering and Touschek decay rates deduced from a fit to the data. In the limit of zero current, the gas scattering rate approaches a finite value, while the Touschek rate vanishes as expected. At $80 \mathrm{~mA}$, the increase of the Touschek rate is apparently halted by instabilities. The respective lifetime contributions are listed in table 2 for $10 \mathrm{~mA}$ and $100 \mathrm{~mA}$.

As another confirmation of the Touschek effect, the bottom part of figure 1 shows data for $22 \mathrm{~mA}$ in 60 bunches (FWHM of the bunch train) and in 15 bunches. The increased bunch current is indeed reflected by a stronger Touschek contribution.
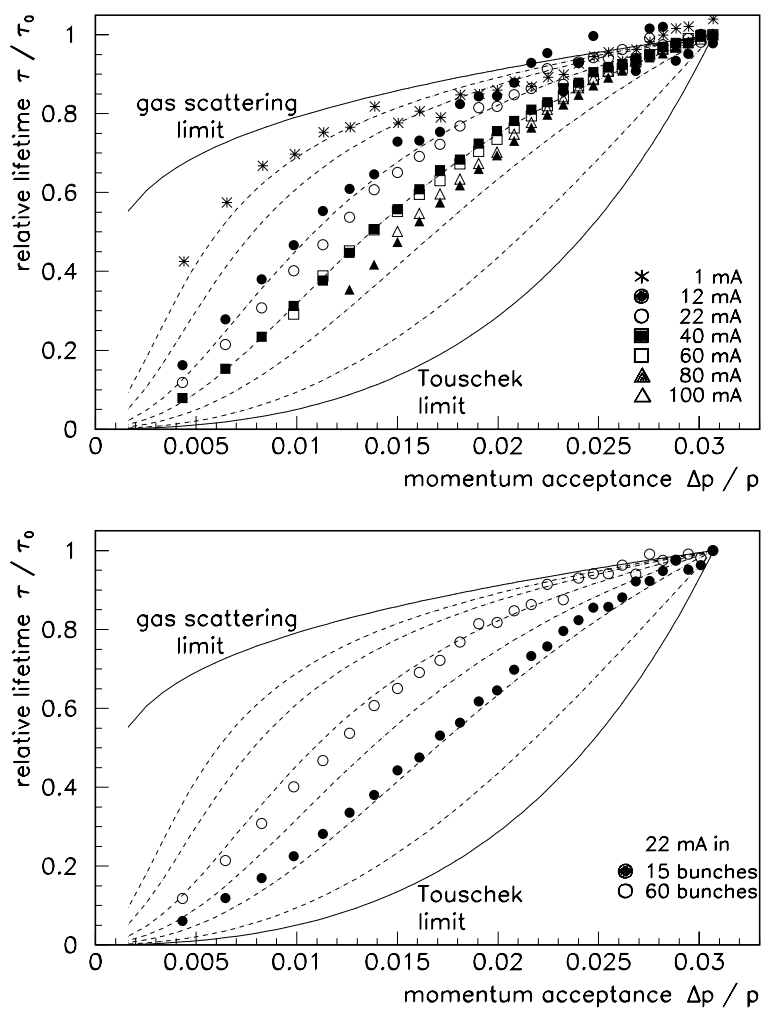

Figure 1: Beam lifetime (normalized to the value $\tau_{0}$ at $\Delta p / p=0.03$ ) as function of the momentum acceptance.

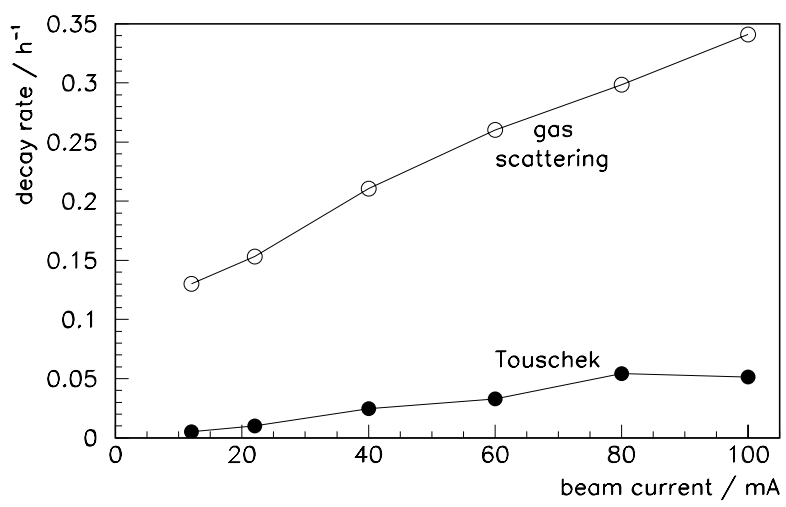

Figure 2: Fitted decay rates versus beam current.

\subsection{Beam Lifetime versus Aperture}

Moving vertical scrapers towards the beam increases the losses from Coulomb scattering (equation 3). With horizontal oscillations coupling into the vertical plane and the presence of vertical dispersion, a vertical scraper $\leq 1 \mathrm{~mm}$ from beam center would also limit the momentum acceptance, but the decay rate from this process is negligible. The quantum lifetime, important below 7-8 $\sigma(\leq 0.1 \mathrm{~mm})$, can be ignored as well.

If dominated by Coulomb scattering, the lifetime would depend quadratically on the scraper position. This is shown in figure 3 (dashed line) together with measured data and 
fits (solid lines) assuming scraper-independent contributions from Bremsstrahlung and Touschek scattering. The fit parameters were the central position of the beam, the aperture $a$, and the Touschek rate, while the Bremsstrahlung rate was kept constant. Figure 3 (top) shows measurements at $10 \mathrm{~mA}$ and $100 \mathrm{~mA}$. The fact that the fit describes the $10 \mathrm{~mA}$ data well confirms the theoretical Bremsstrahlung rate. At larger currents, the inclusion of a small Touschek contribution, increasing with current, clearly improves the fit. As a further confirmation, the Touschek rate was boosted by reducing the rf voltage. Figure 3 (bottom) shows a fit to these data, which agrees well with the expectation. The small deviation at 3-4 $\mathrm{mm}$ is not yet understood.

The undulator chambers with $8 \mathrm{~mm}$ half-height are presumed to be the limiting apertures, whereas the fits yield $a=6.2-6.4 \mathrm{~mm}$ at the undulator entrance. A better agreement cannot be expected since equation 3 is based on purely linear beam optics. Furthermore, the beam may be not exactly on the chamber axis, the dynamic aperture may be smaller than the physical aperture, and the beam cross section may be tilted due to coupling.

\section{CONCLUSIONS AND OUTLOOK}

The BESSY II beam lifetime is consistent with the assumption of residual gas scattering and Touschek scattering as limiting effects. At the time of the experiment, the lifetime was $10 \mathrm{~h}$ at low current, corresponding to an average $\mathrm{N}_{2}$-equivalent pressure of $4 \cdot 10^{-9} \mathrm{hPa}$. A further improvement of the vacuum by an order of magnitude can be expected, and the lifetime will be ultimately limited by Touschek scattering. The Touschek lifetime was found to be $20 \mathrm{~h}$ at $100 \mathrm{~mA}$ in 60 bunches. The measured horizontalvertical coupling was $1.9 \%$. For this case, calculations using the code ZAP [7] suggest a bunch length of $8 \mathrm{~mm}$ and a Touschek lifetime of $15 \mathrm{~h}$.

Larger beam currents, improvement of the coupling to well below $1 \%$, and the operation of feedback systems against longitudinal and transverse multibunch instabilities [8] will certainly increase the Touschek rate. On the other hand, the bunch current can be reduced by filling more buckets $(\sim 300)$. Furthermore, the design of passive third-harmonic rf cavities to increase the bunch length is in progress.

Table 2: Contribution of different lifetime limiting effects.

\begin{tabular}{|l|r|r|}
\hline & $10 \mathrm{~mA}$ & $100 \mathrm{~mA}$ \\
\hline elastic scattering on nuclei & $18 \mathrm{~h}$ & $6.8 \mathrm{~h}$ \\
inelastic scattering on nuclei & $19 \mathrm{~h}$ & $7.2 \mathrm{~h}$ \\
elastic scattering on electrons & $1100 \mathrm{~h}$ & $420 \mathrm{~h}$ \\
inelastic scattering on electrons & $53 \mathrm{~h}$ & $20 \mathrm{~h}$ \\
Touschek effect & $190 \mathrm{~h}$ & $20 \mathrm{~h}$ \\
\hline combined lifetime & $7.4 \mathrm{~h}$ & $2.6 \mathrm{~h}$ \\
\hline
\end{tabular}
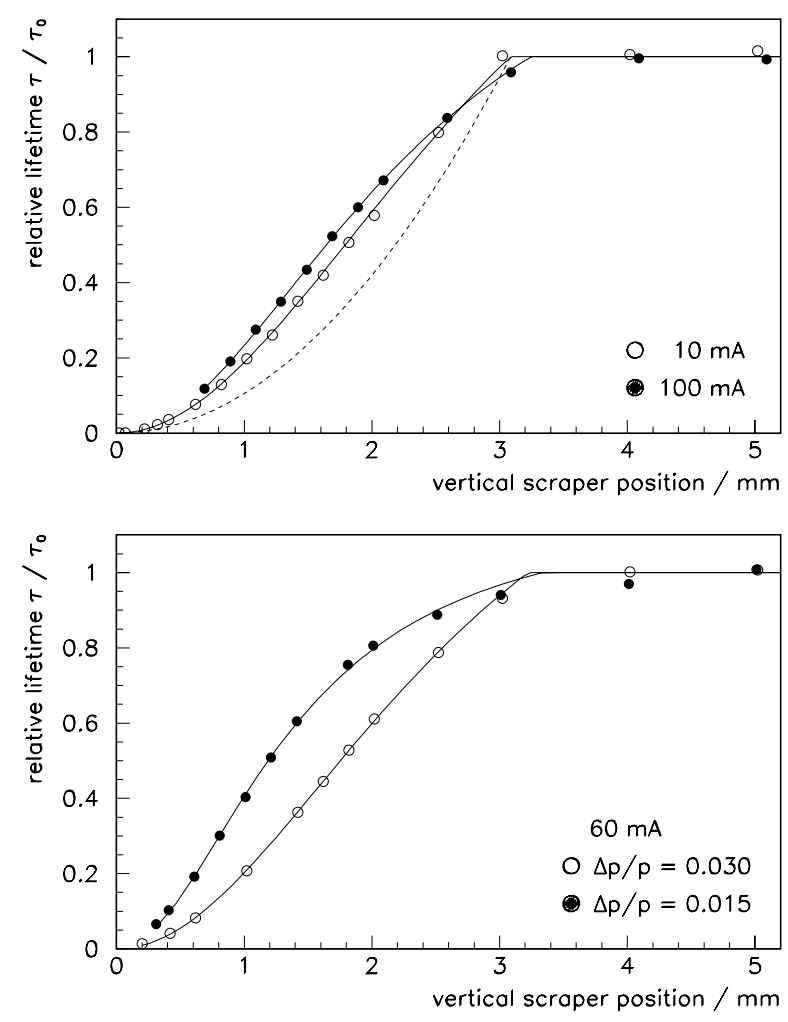

Figure 3: Beam lifetime (normalized to the retracted scraper value $\tau_{0}$ ) versus vertical scraper position.

\section{ACKNOWLEDGEMENTS}

The author would like to thank the BESSY II commissioning team whose combined effort made these measurements possible.

\section{REFERENCES}

[1] R.P. Walker et al.: 'Beam Lifetime in ELETTRA', Proceedings of the 4th European Particle Accelerator Conference, London (1994), p.1347.

[2] J. Byrd, W. Decking, C. Kim, D. Robin: 'Lifetime Studies at the Advanced Light Source', Proceedings of the 6th European Particle Accelerator Conference, Stockholm (1998), p.1347.

[3] R. Bakker for the BESSY II Project Team: 'Status and Commissioning Results of BESSY II', this conference.

[4] J. le Duff: 'Current and Current Density Limitations in Existing Electron Storage Rings', NIM A239 (1985), p.83, and references therein.

[5] S. Khan: 'Simulation of the Touschek Effect for BESSY II A Monte Carlo Approach', Proceedings of the 4th European Particle Accelerator Conference, London (1994), p.1192.

[6] The photodiode signal was made available by R. Thornagel et al. (Physikalisch-Technische Bundesanstalt, Berlin).

[7] M. Zisman, S. Chattopadhyay, J. Bisognano: 'ZAP User's Manual', LBL-21270 (1986).

[8] S. Khan, T. Knuth: 'BESSY II Feedback Systems', this conference. 